\title{
The compressible turbulent shear layer: an experimental study
}

\author{
By DIMITRI PAPAMOSCHOU AND ANATOL ROSHKO \\ Graduate Aeronautical Laboratories, California Institute of Technology, \\ Pasadena, CA 91125 , USA
}

(Received 2 September 1987 and in revised form 20 May 1988)

The growth rate and turbulent structure of the compressible, plane shear layer are investigated experimentally in a novel facility. In this facility, it is possible to flow similar or dissimilar gases of different densities and to select different Mach numbers for each stream. Ten combinations of gases and Mach numbers are studied in which the free-stream Mach numbers range from 0.2 to 4 . Schlieren photography of 20 -ns exposure time reveals very low spreading rates and large-scale structures. The growth of the turbulent region is defined by means of Pitot-pressure profiles measured at several streamwise locations. A compressibility-effect parameter is defined that correlates and unifies the experimental results. It is the Mach number in a coordinate system convecting with the velocity of the dominant waves and structures of the shear layer, called here the convective Mach number. It happens to have nearly the same value for each stream. In the current experiments, it ranges from 0 to 1.9. The correlations of the growth rate with convective Mach number fall approximately onto one curve when the growth rate is normalized by its incompressible value at the same velocity and density ratios. The normalized growth rate, which is unity for incompressible flow, decreases rapidly with increasing convective Mach number, reaching an asymptotic vaue of about 0.2 for supersonic convective Mach numbers.

\section{Introduction}

As a result of today's increasing importance of supersonic combustion, there has recently been renewed interest in the compressible turbulent shear layer. Compressibility plays a crucial role in the stability and mixing of shear layers, its effects being capable of producing order-of-magnitude changes compared with incompressible flow. It is essential that these effects be understood in a coherent and universal way. The limited amount of experimental data in two-stream compressible turbulent shear layers indicates the need for extensive experimental investigations where parametric studies of such flows can be carried out.

It has been observed that turbulent shear layers with one stream supersonic and one stream at rest (e.g. at the edges of jets) spread more slowly than incompressible shear layers. Birch \& Eggers (1973) have compiled a survey of such experiments. Although some inconsistencies exist among the various investigators, there is a definite trend of decreasing grow th rate with increasing free-stream Mach number. In most one-stream experiments, increasing Mach number is accompanied by decreasing temperature and thus increasing density of the jet. The thinning of the shear layer with increasing Mach number was thus attributed by many investigators to the density ratio between the jet and the external gas. This led to models incorporating 
Howarth-Dorodnitsyn-type transformations, density-dependent eddy viscosities, etc.

Whether density effects alone could account for the differences in spreading rate was one of the questions that motivated the experiment of Brown \& Roshko (1974), who built an apparatus in which incompressible shear layers with large density differences could be studied by using different gas combinations, like helium and nitrogen, at low speeds. It was found that, although there is some effect of density on the spreading rate, it is very much smaller than what is observed in the supersonic case. It was therefore concluded that compressibility, per se, affects in a fundamental way the development of the supersonic shear layer.

Little information about turbulent growth rates can be extracted from the scarce two-stream experiments in the literature, primarily because most measurements were taken in the near field of a flow with a strong wake component (Bailey \& Kuethe 1957). Ortwerth \& Shine (1977) conducted an interesting heterogeneous flow experiment, mentioned later in this section, but did not provide clear growth-rate data. More recently, Chinzei et al. (1986) generated turbulent shear layers with air in both streams, where one Mach number is supersonic and the other ranges from subsonic to supersonic. Unfortunately, their growth-rate measurements are hard to interpret quantitatively because they are based on an uncommon definition of shearlayer thickness and are correlated ignoring density effects. Other two-stream experiments concentrate on transition (Shackleford et al. 1973; Demetriades \& Brower 1982) and ejector performance (Dutton, Mikkelsen \& Aiddy 1982).

Perhaps the most elementary feature that distinguishes a supersonic flow from a subsonic one is that in the supersonic case a disturbance does not propagate upstream and remains confined within a Mach cone, while in the subsonic case a disturbance is felt throughout the flow field. Given the limited region of influence of a supersonic disturbance, we might expect that a supersonic shear layer is more stable, therefore mixes more slowly, than a subsonic one. In converting this abstract notion to a more concrete one, the question arises: in what frame of reference do we measure the compressibility of the shear layer? In the case of the one-stream shear layer, we might choose the laboratory frame of reference. In the case of the twostream shear layer, however, this becomes a very poor choice. As an extreme example, consider a shear layer comprising two supersonic streams with nearly equal velocities. Clearly, that shear layer should not be described as intrinsically supersonic when the velocity difference between the free streams is low subsonic. The problem of finding the proper frame of reference becomes even more complex when the gases have different speeds of sound. Nevertheless, it is essential that a sensible choice for a frame of reference be made in which the intrinsic compressibility of the flow is properly characterized.

In two-stream compressible shear layers, the effects of compressibility will be coupled not only with the effects of density ratio, but also with those of velocity ratio. The spreading rate of the incompressible turbulent shear layer has been found to depend largely on these two parameters, most importantly on velocity ratio. To understand and quantify the effects of compressibility, a scheme must be invented by means of which compressibility effects are somehow uncoupled from those of the other relevant parameters. Useful comparisons can only be made when one parameter is varied at a time, something that is lacking in the correlations performed by previous experimental investigators.

Subsonic turbulent shear layers contain large, so-called 'coherent' structures reminiscent of those in the early stages of instability. It was realized that the growth 


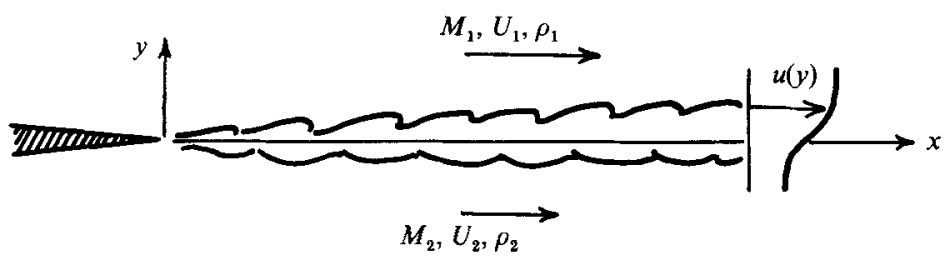

Figure 1. Shear-layer geometry.

of the shear layer is governed primarily by its instability at these large scales. There is already visual evidence that large-seale structures exist in supersonic shear layers. This evidence comes from the experiments of Ortwerth \& Shine (1977), where a stream of helium at $M=3$ mixes with a stream of nitrogen also at $M=3$, and of Oertel (1979) at the edges of supersonic jets. Not much is presently known about the nature of these structures nor to what extent they dominate the mixing process in the compressible case. If their role is important, then the question as to whether they are two- or three-dimensional, spanwise-coherent or oblique, becomes important and is still unresolved.

The objective of the present study is to investigate turbulent two-stream shear layers between similar or dissimilar gases at a variety of free stream Mach numbers, ranging from subsonic to high supersonic. In that way, compressibility effects, together with those of density and velocity ratios, are studied. The general configuration of the shear layer and the variables involved are summarized in figure 1. The current investigation focuses on the measurement of turbulent growth rates from Pitot-pressure surveys. Such measurements are performed at 10 Mach number-gas combinations with varying degrees of compressibility, so that statistically meaningful trends are established. Considerable effort is made to correlate and unify the results, using ideas stated earlier, so that the net effect of compressibility can be abstracted as clearly as possible. Additionally, instantaneous flow visualization shows the remarkably small spreading rates and indicates the existence of large-scale structures.

\section{Apparatus and instrumentation}

It was our intention to create and study shear layers between gases of variable density, velocity and Mach number. A new facility that meets our objectives was designed and built. It is shown schematically on figure 2. Basically, it is a twostream, blow-down supersonic wind tunnel with two independent supply sides. Each supply side is connected to a manifold of gas bottles initially at 2000 psi. Large-flowrate pressure regulators (Grove 202G) reduce the gas pressure to a range from 15 to 100 psia. Each gas enters the settling chamber by means of a fast-acting solenoid valve (ASCO) which starts and stops the flow. The downstream end of the flow channel is connected to a low-pressure tank, evacuated by a vacuum pump of large displacement (Beach-Russ 325RP). The operation of the facility is intermittent.

After passing through flow-management devices located on the settling chamber, each gas is expanded to its design Mach number by means of a contoured centrebody. When placed between the upper and lower test-section walls, the centrebody forms two supersonic nozzles. The nozzle contours are calculated by the method of characteristics and produce nearly uniform exit conditions. The two gases come into contact downstream of the centrebody's trailing edge. The centrebody is replaceable; thus a number of them are used for varying the Mach number-specific heat ratio 


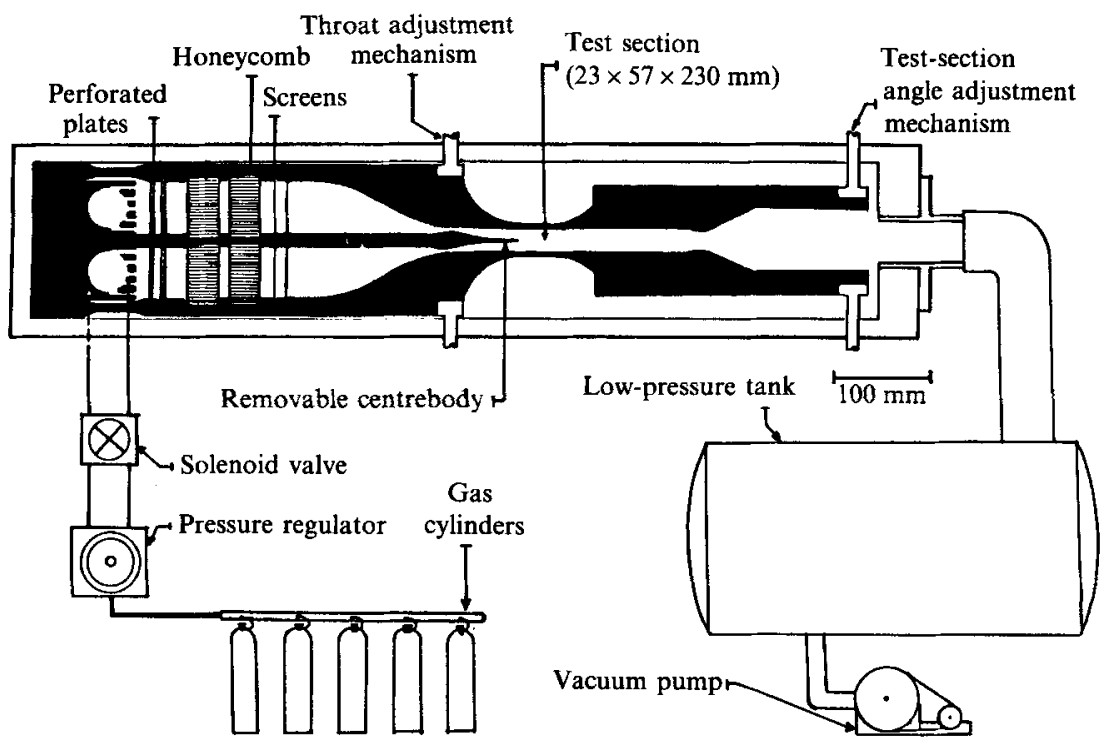

Figure 2. Schematic of flow facility.

combination. The centrebody lengths, from sonic throat to trailing edge, range from $50 \mathrm{~mm}$ to $61 \mathrm{~mm}$. The thickness of the trailing edge is $0.3 \mathrm{~mm}$.

The test section is $23 \mathrm{~mm}$ high (at the nozzle exit), $57 \mathrm{~mm}$ wide and $230 \mathrm{~mm}$ long. Optical-quality glass windows extend from the nozzle throat to the end of the test section. To minimize streamwise pressure gradients, the angles of the upper and lower walls are adjustable to within $\pm 3^{\circ}$. In all present experiments, the walls were set to diverge at an angle between $1^{\circ}$ and $2^{\circ}$.

A schlieren optical system, shown on figure 3 , provides instantaneous flow visualization. It is a conventional one, although folded to conserve space, with the knife edge parallel to the flow direction. Illumination is provided by a spark source of 20-ns duration (Xenon N-787B). The parallel beam entering the test section has a diameter of $100 \mathrm{~mm}$. The optical components are stationary but the flow channel traverses on rails to allow photography of every part of the test section.

A small Pitot probe, flattened at the inlet, traverses the shear layer at any desired streamwise location (figure 4 ). It pivots at constant speed around a support located downstream of the test section. Its incidence angle to the flow never exceeds $10^{\circ}$. The temporal and spatial resolutions that it offers are satisfactory for our operation. The shear-layer Pitot thickness, $\delta_{\text {pit }}$, is the width of the Pitot-pressure profile from $5 \%$ to $95 \%$ of the difference of the free-stream values. If the profile has a wake-like defect, it is split into a top and bottom part at the location of the minimum; $\delta_{\text {pit }}$ is then measured from $95 \%$ of the bottom difference with respect to the minimum, to $95 \%$ of the top difference. These definitions of $\delta_{\text {pit }}$ are illustrated on figure 5. A similar definition of thickness, based on the velocity profile, has been used by Spencer \& Jones (1971). We must point out that a defect in the Pitot-pressure profile does not necessarily imply a wake component in the velocity, but may rather be connected to dissipation in the mixing region.

Twelve static-pressure ports along the test section (6 on the top wall and 6 on the bottom wall), as well as total-pressure ports in the settling chamber, are connected to pressure transducers (Setra Systems 204) via a Scanivalve system. The outputs of the transducers are recorded on a digital oscilloscope (Nicolet 206) and stored on 


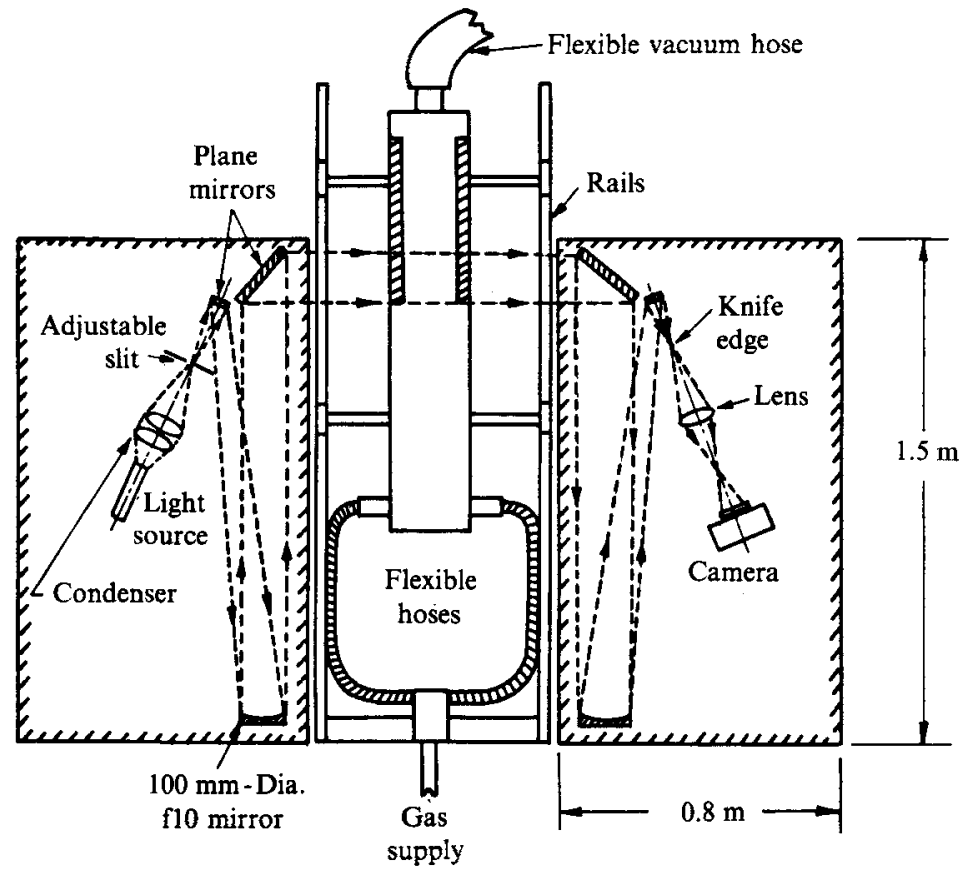

Figure 3. Schematic of schlieren optical system.

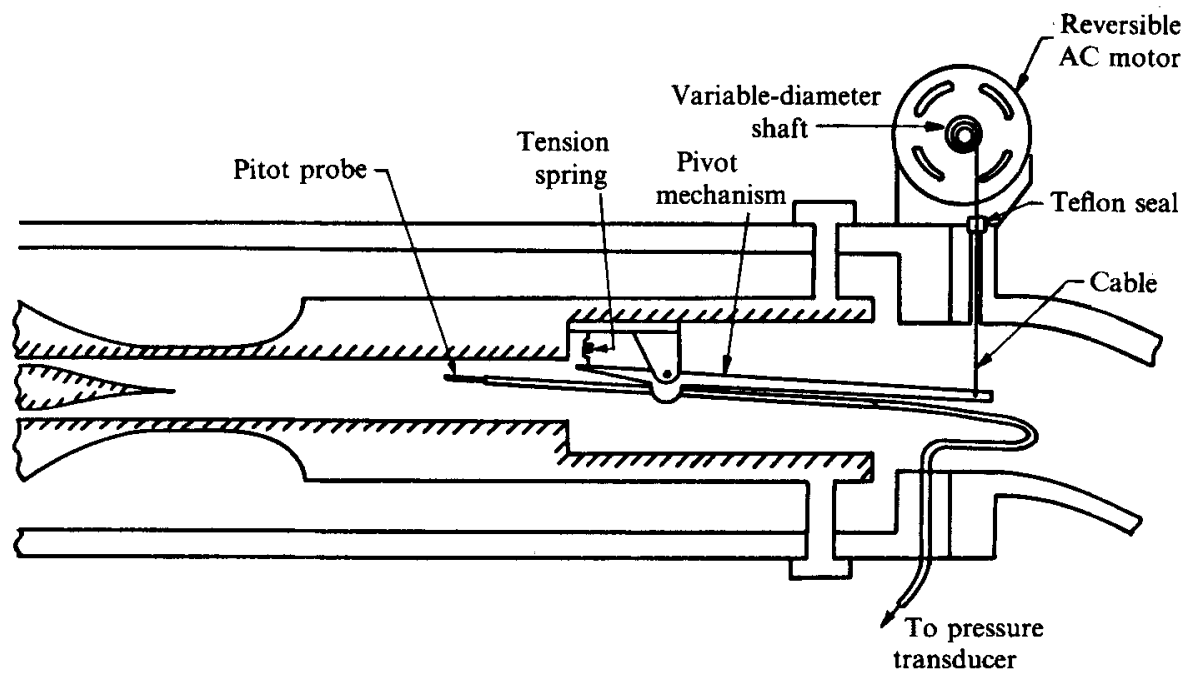

Figurfi 4. Pitot-probe mechanism.

floppy disc. The total temperatures in the settling chamber are also recorded, although their values do not vary significantly from room values. A series of mechanical pressure gauges, connected to every pressure stage of the facility, help monitor the operation and set the regulator pressures.

The starting and stopping of the flow, photography, probe traversing, and recording are done automatically with an Intel 8085 microprocessor connected to a system of relays. Each schlieren picture and Pitot-pressure profile is accompanied by a record of the static and total pressures that occurred during the run. 


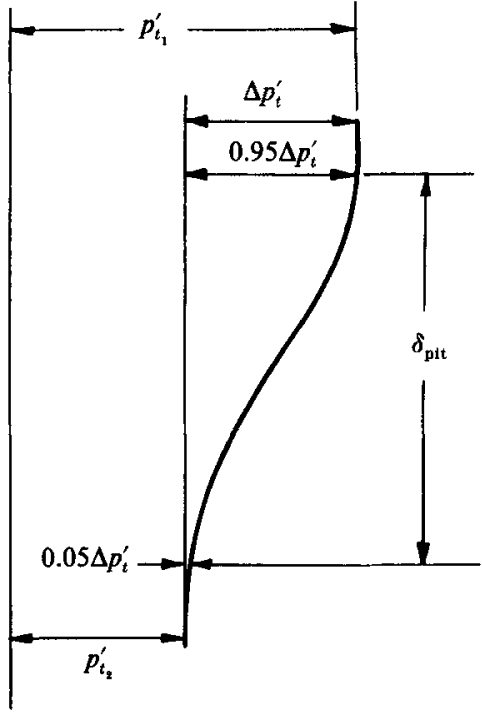

(a)

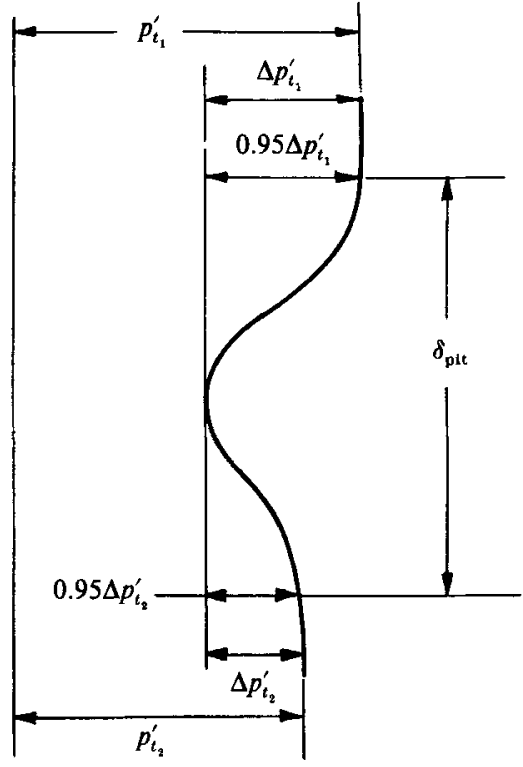

(b)

Figure 5. Definition of Pitot thickness, $\delta_{\mathrm{pit}}$. (a) Monotonic Pitot-pressure profile; $(b)$ profile with defect.

\section{Run conditions}

Ten combinations of Mach numbers and gases are investigated in the current experiments. The static pressure in the test section is of the order of 1 psia, while the total pressures vary from 15 to 80 psia, depending primarily on the nozzle-exit Mach number. Each run is restricted to 1.5 to $2.0 \mathrm{~s}$ duration to conserve the bottled gases.

For each Mach number-gas combination, the total pressures are set such that the static pressures of the two free streams at the nozzle exits are nearly equal. This is done to avoid strong compression and expansion waves emanating from the trailing edge. One might expect that if the pressures were exactly matched, the flow would be free of waves throughout the test section. Nevertheless, the displacement thicknesses of the trailing-edge boundary layers, as well as the thickness of the trailing edge itself, make waves unavoidable. Furthermore, the displacement thickness of the shear layer produces further waves and complicates the flow field significantly.

To diminish the strength of these waves to a minimum, the total pressures (which are directly proportional to their respective nozzle-exit static pressures) are manipulated so that the static-pressure distribution along the test section is as smooth as possible (as already mentioned, the pressure distribution is monitored by the use of pressure taps along the test-section walls). This procedure results in the trailing-edge static pressures being close, but not always exactly equal. The pressuresetting operation constituted the major difficulty of this experiment because it required numerous trials which expended time and gas. Compounding the difficulty were the occasionally erratic outputs of the pressure regulators and the adverse pressure gradient discussed below.

In two cases, the slow stream is made low subsonic by reducing its total pressure 


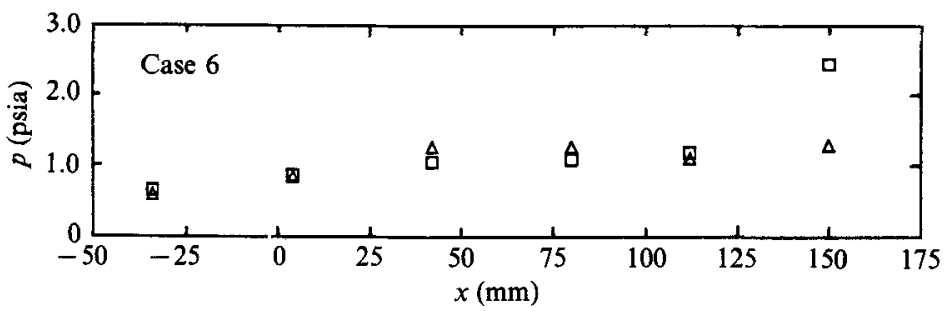

Frgure 6. Typical wall pressure distribution. Squares, upper wall; triangles, lower wall.

so that it is close to the desired test-section static pressure. The supersonic nozzle now acts as a slowly diverging subsonic diffuser (only the lowest-Mach number nozzle is used in this fashion). In these cases, the above-described pressure-setting procedure becomes ineffective because the static pressure throughout the subsonic stream adjusts itself to the highest one occurring in the supersonic stream.

The measured static-pressure distribution along the test-section walls surprised us at first. In all our cases, the static pressure rises rapidly near the trailing edge, then levels off in the far field. The pressure rise, relative to the trailing-edge value, is $20 \%$ to $50 \%$ depending on the test case. A typical pressure trace is shown on figure 6 where we see an additional sharp pressure rise at the very end of the test section. We believe that this is the result of a localized upstream influence of the diffuser section, rather than pressure recovery associated with the shear layer itself.

It came to our knowledge that the phenomenon of pressure recovery in supersonic shear layers has been observed before, most dramatically in the supersonic-ejector experiments of Dutton et al.(1982). There, it was found that the compressible mixing layer in a constant-area duct exhibits a strong pressure recovery, much like in a supersonic diffuser. In a general sense, we may attribute the cause of this pressure rise to increased entropy due to mixing. In supersonic flows, entropy increase leads to increase of pressure together with decrease of total pressure and Mach number. An equivalent, more mechanical interpretation is that the pressure rise is a result of the shear-layer displacement thickness which, in our cases, appears to act as a solid wedge inserted between the two streams.

The divergence of the test-section walls was instrumental in maintaining a fairly constant static pressure for the downstream two-thirds of the test section. The growth-rate measurements were performed in the constant-pressure region, so we believe that they are largely unaffected by the initial pressure rise. Consequently, the Mach number in that region, $M$, is lower than the ideal nozzle-exit Mach number $M_{\mathrm{e}}$. Using the measured values of the local free stream-Pitot and static pressures, $M$ is calculated using the Rayleigh Pitot formula.

In table 1 that follows, the ideal and measured Mach number-gas combinations are listed together with their density ratios $\rho_{2} / \rho_{1}$ and velocity ratios $U_{2} / U_{1}$. Subscripts 1 and 2 indicate the high-speed and low-speed side respectively. $M_{\mathrm{c}_{1}}$ is a compressibility-effect parameter to be defined later.

The unit Reynolds number is of the order of $10000 \mathrm{~mm}^{-1}$. At the conditions described above, the trailing-edge boundary layers are laminar. Their momentum thickness, $\theta$, is calculated by a compressible Thwaites method (Rott \& Crabtree 1952). A typical value for the high-speed $\theta$ is $0.05 \mathrm{~mm}$. The very large supersonic velocities render buoyancy effects negligible. Richardson numbers (defined as $-g \Delta \rho \delta_{\mathrm{pit}} /(\bar{\rho} \Delta U)$, where $\bar{\rho}$ is the mean density) are of the order of $10^{-7}$. 


\begin{tabular}{cccccccccc}
\hline Case & Gas 1 & $M_{\mathrm{e}_{1}}$ & $M_{1}$ & Gas 2 & $M_{\mathrm{e}_{2}}$ & $M_{2}$ & $\rho_{2} / \rho_{1}$ & $U_{2} / U_{1}$ & $M_{\mathrm{c}_{1}}$ \\
1 & $\mathrm{~N}_{2}$ & 1.9 & 1.6 & $\mathrm{Ar}$ & 4.0 & 3.3 & 4.4 & 0.93 & 0.07 \\
2 & $\mathrm{Ar}$ & 4.0 & 3.4 & $\mathrm{Ar}$ & 2.0 & 1.8 & 0.43 & 0.81 & 0.26 \\
3 & $\mathrm{~N}_{2}$ & 3.3 & 3.1 & $\mathrm{~N}_{2}$ & 1.9 & 1.7 & 0.54 & 0.74 & 0.33 \\
4 & $\mathrm{~N}_{2}$ & 3.0 & 2.8 & $\mathrm{Ar}$ & 3.0 & 2.6 & 1.8 & 0.75 & 0.39 \\
5 & $\mathrm{~N}_{2}$ & 3.5 & 3.2 & $\mathrm{Ar}$ & 2.3 & 2.1 & 1.2 & 0.67 & 0.55 \\
6 & $\mathrm{He}$ & 2.3 & 1.7 & $\mathrm{~N}_{2}$ & 3.5 & 3.0 & 9.2 & 0.52 & 0.64 \\
7 & $\mathrm{Ar}$ & 4.0 & 3.1 & $\mathrm{Ar}$ & 0.2 & 0.2 & 0.24 & 0.13 & 0.89 \\
8 & $\mathrm{He}$ & 3.0 & 2.6 & $\mathrm{~N}_{2}$ & 3.0 & 2.8 & 5.5 & 0.42 & 1.05 \\
9 & $\mathrm{He}$ & 4.0 & 3.4 & $\mathrm{~N}_{2}$ & 1.9 & 1.6 & 2.2 & 0.29 & 1.44 \\
10 & $\mathrm{He}$ & 4.0 & 3.1 & $\mathrm{Ar}$ & 0.3 & 0.3 & 2.4 & 0.04 & 1.81
\end{tabular}

TABLE 1. Ideal and measured Mach number-gas combinations, with their density and velocity ratios

\section{Flow pictures}

Schlieren photographs of eight cases are shown on figure $7(a-g)$. Cases 2,5 and 10 are omitted because they did not produce good flow visualization. For each case, two photographs are shown: the upper one covers the upstream part of the test section and the lower one covers the downstream part. They are taken at different runs under the same flow conditions and overlap slightly. The trailing edge of the centrebody is located in the upstream pictures where the waves originate. In all eight cases, the shear layer is seen to become turbulent immediately downstream of the trailing edge.

The first striking feature of the pictures is the unusually small spreading rate of the shear layers. Although the visual thickness, as it appears on the picture, depends to some extent on the sensitivity of the schlieren system, it is unquestionable that the present shear layers grow at a fraction of the usually observed subsonic rate. The small growth ratc is not necessarily an effect of compressibility alone. Other important parameters, namely the velocity and density ratios, also govern the growth rate. Later in this report we try to distinguish the compressibility effects from those of the other parameters.

The other striking feature is the presence of large-scale structures. Here the term 'large-scale' is used with reference to the local thickness of the layer. Such structures have been observed in the past in supersonic jets (Oertel 1979) and shear layers (Ortwerth \& Shine 1977) but in limited flow regimes. The present pictures establish with little doubt the existence of large structures in compressible shear layers under a large varicty of conditions.

Earlier in our research programme, we inferred visual growth rates from the pictures (Papamoschou \& Roshko 1986). The results are subjective and of limited accuracy, but agree qualitatively with the present ones.

\section{Growth rates}

Figure 8 shows representative Pitot-pressure surveys. The Pitot-thickness measurements are extracted from these profiles by the method outlined in $\S 2$. The turbulent growth rate is inferred from at least four Pitot-thickness data points in the far field, further than $75 \mathrm{~mm}$ from the trailing edge. In that region, $x / \theta_{1}>1000$ for all cases. At first thought, this seems to satisfy Bradshaw's (1966) criterion for fully 
(a)

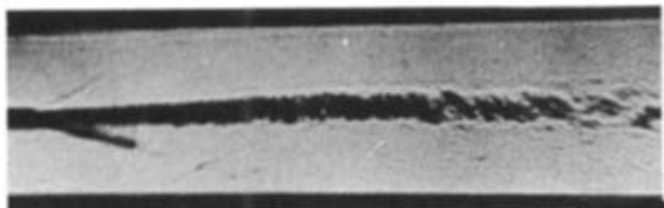

$$
\frac{U_{2}}{U_{1}}=0.93 \quad \frac{\rho_{2}}{\rho_{1}}=4.4
$$$$
M_{\epsilon_{1}}=0.07
$$

Case 1

$\mathrm{N}_{2}$ @ $M_{1}=1.6$

$\mathrm{Ar} @ M_{2}=3.3$ (b)

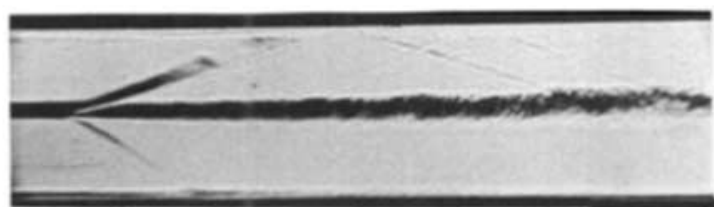

$$
\frac{U_{3}}{U_{1}}=0.74 \quad \frac{\rho_{2}}{\rho_{1}}=0.54
$$

$M_{\epsilon_{3}}=0.33$

(c)

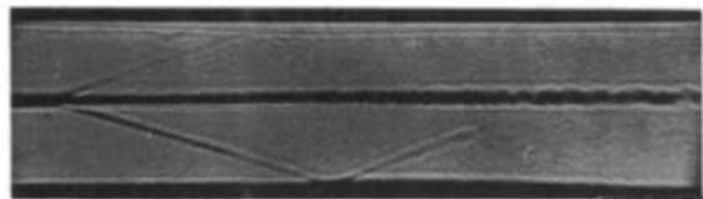

Case 3

$\mathrm{N}_{2} @ M_{1}=3.1$

$\mathrm{N}_{2} @ M_{2}=1.7$ (d)

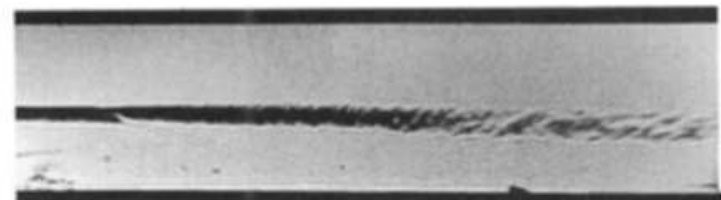

Case 4

$\mathrm{N}_{2} @ M_{1}=2.8$

Ar $@ M_{z}=2.6$

Case 6

He a $M_{1}=1.7$

$\mathrm{N}_{2}$ \& $M_{z}=3.0$

$$
\begin{gathered}
\frac{U_{3}}{U_{1}}=0.52 \quad \frac{\rho_{2}}{\rho_{1}}=9.2 \\
M_{\epsilon_{1}}=0.64
\end{gathered}
$$

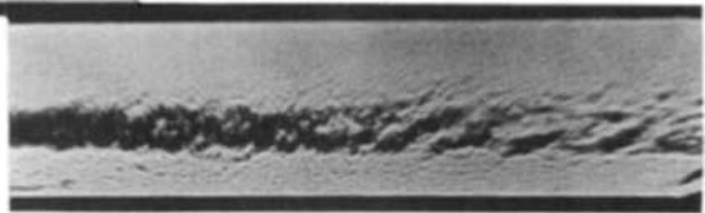

Figure $7(a-d)$. For caption see next page. 
(e)

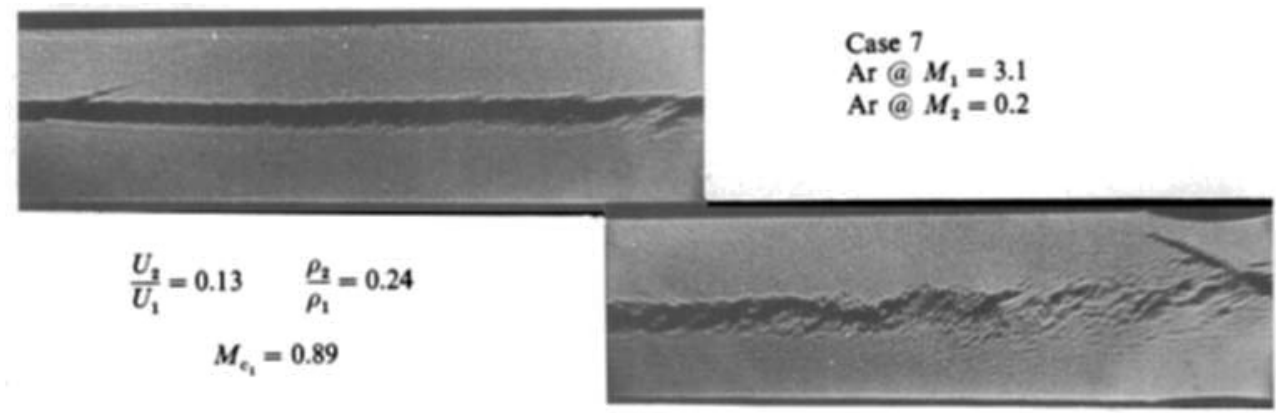

$(f)$

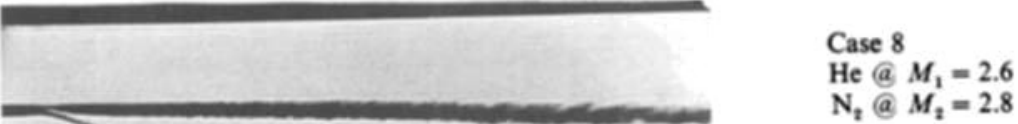

(g)

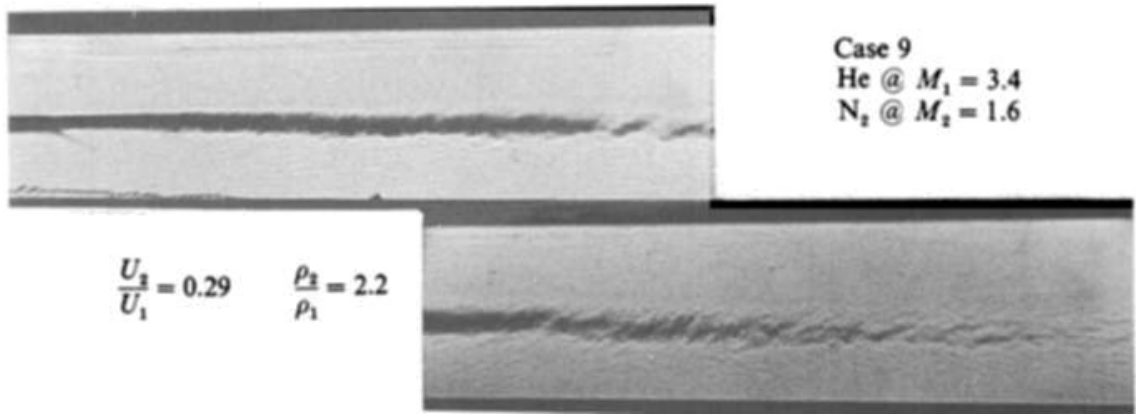

Figure 7. Schlieren photographs. (a) Case $1 ;(b)$ Case $3 ;(c)$ Case $4 ;(d)$ Case $6 ;(e)$ Case $7 ;(f)$ Case 8; $(g)$ Case 9. Mach number-gas combination and velocity and density ratios are given in table 1 .

developed turbulence. It must be kept in mind, however, that Bradshaw's results are based on one-stream, incompressible shear-layers with uniform density and thus may not be as valid for the flows we are considering. It is especially obvious that Bradshaw's criterion will lose its validity when $U_{2} / U_{1}$ is close to unity. Possibly a more pertinent parameter to use in that case is $x_{\text {eff }} / \theta_{1}$, where $x_{\text {eff }}=x\left(1-U_{2} / U_{1}\right)$. Excluding Case 1, which is a wake flow, all other cases satisfy $x_{\text {eff }} / \theta_{1}>500$ in the far field.

Given that the shear layer is fully turbulent, we expect it to grow linearly. With that in mind, a straight line is fitted through the downstream Pitot-thickness data by the method of least squares. Case 1 is omitted from this procedure because it is a wake flow, therefore is not expected to grow linearly. Figure 9 shows the fit to the data. The shear layers appear indeed to grow linearly. The standard deviation of the data from the least-squares fit, expressed as a growth-rate error, does not exceed $5 \%$. The accuracy of the thickness measurement itself is within $\pm 5 \%$. The overall accuracy of the growth-rate measurement is thus estimated to be roughly $\pm 10 \%$. 

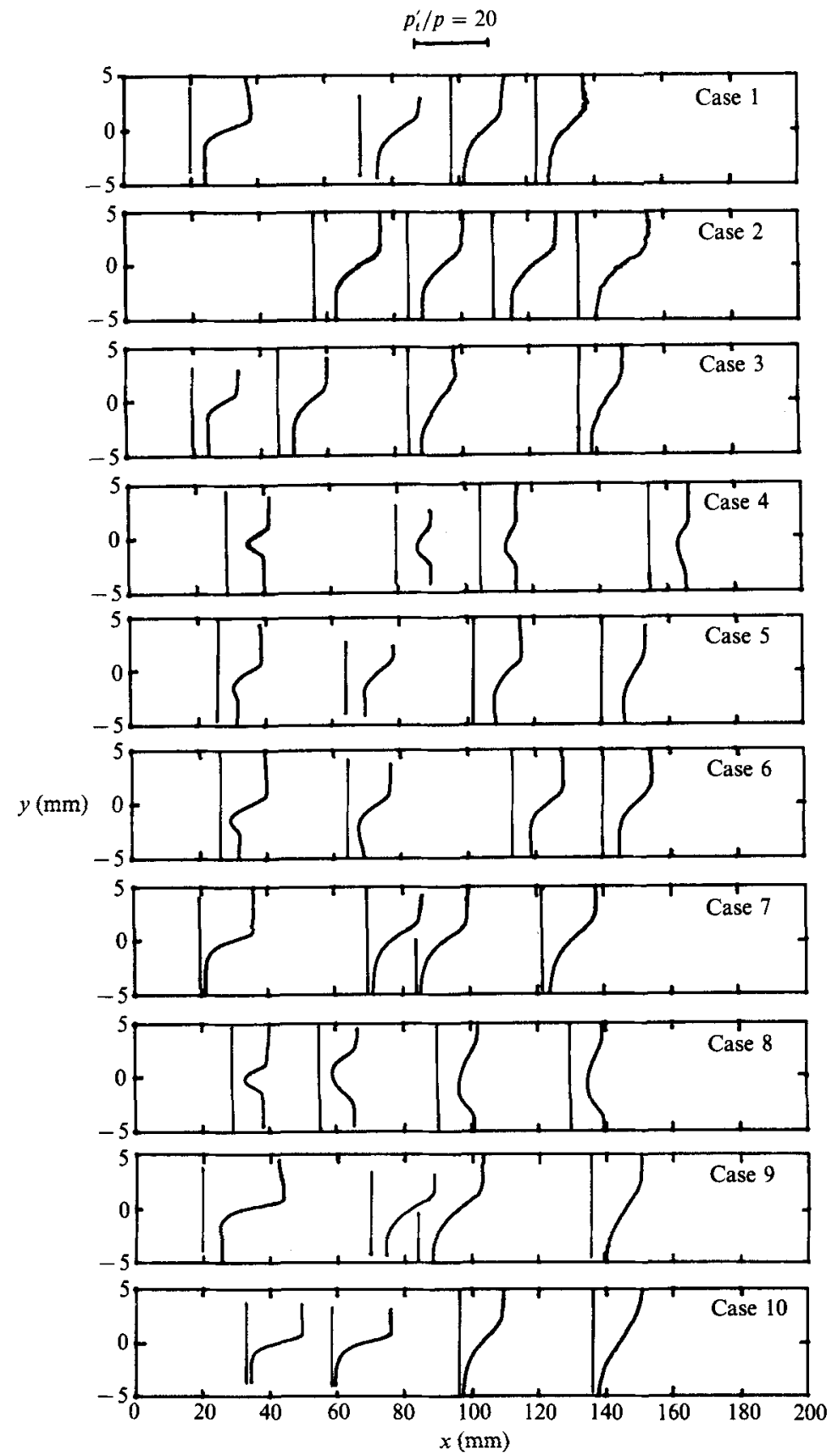

Figure 8. Selected Pitot-pressure profiles. Pitot pressure $\left(p_{t}^{\prime}\right)$ is normalized by local static pressure $(p)$.

In $\$ 7$ important growth-rate comparisons are made between the present compressible flows and incompressible results from the literature. For that, there is a need to establish subsonic relations among the thickness measured here, $\delta_{\text {pit }}$, and thicknesses widely used in the literature, namely the visual thickness $\delta_{\text {vis }}$ and the vorticity thickness $\delta_{\omega \omega}$. For the latter two, it is customary to assume $\delta_{t v}=0.5 \delta_{\mathrm{vis}}$, 


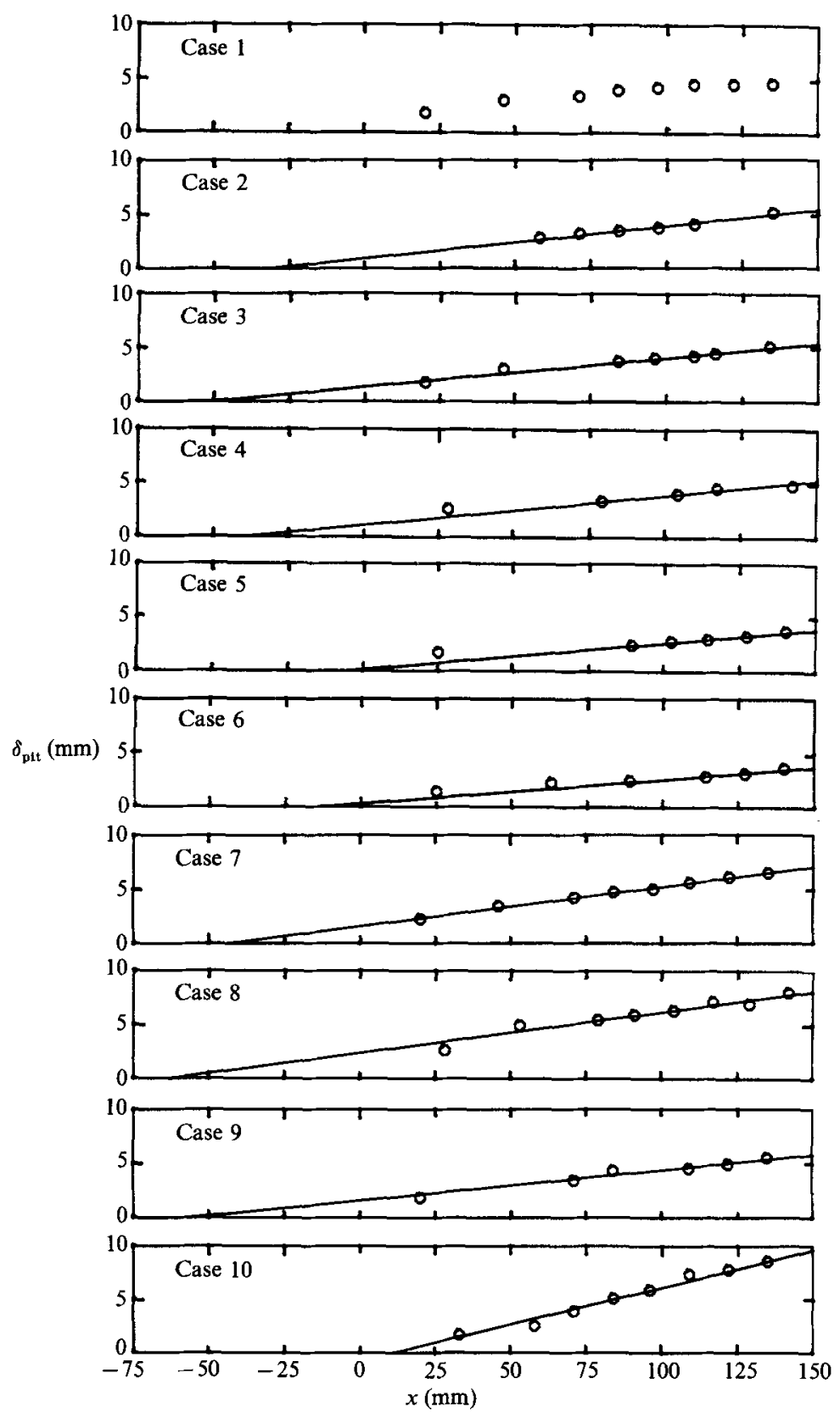

Figure 9. Measurement of Pitot thickness with least-squares, straight-line fit only to data points downstream of $x=75 \mathrm{~mm}$.

although this relation has not been confirmed for variable-density flows. For hyperbolic-tangent velocity profile, $U_{2} / U_{1}=0.5$ (an average value in our flows), and uniform density, $\delta_{\text {pit }} / \delta_{\omega}=1.44$. Consequently, $\delta_{\text {pit }} / \delta_{\text {vis }}=0.72$. We must note that experimental velocity profiles tend to deviate from such simple functions, especially near the edges of the layer (Spencer \& Jones 1971). The ratio $\delta_{\text {pit }} / \delta_{\text {vis }}$ was also deduced from the experimental results of Brown \& Roshko (1974) and of Konrad (1976). In both works, $\delta_{\text {vis }}$ is measured from flow photographs. Figure 11 of Brown 
\& Roshko shows the Pitot-pressure distribution for a flow with $U_{2} / U_{1}=0.38$ and $\rho_{2} / \rho_{1}=7$, from which we obtain $\delta_{\text {pit }} / \delta_{\text {vis }}=0.90$. From Konrad, using the velocity profile for $U_{2} / U_{1}=0.38$ and $\rho_{2} / \rho_{1}=1$ we obtain $\delta_{\text {pit }} / \delta_{\text {vis }}=0.83$. For our purposes, therefore, we choose to assume $\delta_{\text {pit }} / \delta_{\text {vis }}=0.8$, roughly the average of the theoretical and experimental values. Admittedly, there is some arbitrariness in that decision which reflects the scarcity of thickness data and of profile measurements in incompressible heterogeneous shear layers.

\section{A compressibility-correlation parameter}

At this point, we search for meaningful correlations between the growth rate and the flow parameters. Throughout this section and $\$ 7$ our discussion is restricted to two-dimensional disturbances, i.e. disturbances whose direction of propagation is identical to that of the mean flow. This is not to imply that the effects of threedimensional (oblique) disturbances may not be noteworthy or that we ignore their possible importance. On the contrary, such effects may profoundly alter the character of the flow and the analyses that follow. However, our experimental setup did not allow for their detection. We choose to first address the two-dimensional problem which is simpler and which introduces concepts that may later be useful for understanding the more complex three-dimensional case. A brief discussion of the possible effects of three-dimensionalities is presented in $\S 8$.

Since the flow is fully turbulent, it is assumed that Reynolds-number effects on the growth rate are negligible and that the growth rate for perfect gas can be expressed in the form

$$
\delta^{\prime}=f\left(\frac{U_{2}}{U_{1}}, \frac{\rho_{2}}{\rho_{1}}, \frac{\gamma_{2}}{\gamma_{1}}, M_{1}\right)
$$

where $\delta^{\prime}=\mathrm{d} \delta / \mathrm{d} x . M_{2}$ does not appear in (1) because it is redundant: for constant static pressure across the layer, $M_{2}$ is related to $M_{1}$ by

$$
M_{2}=M_{1} \frac{U_{2}}{U_{1}}\left(\frac{\rho_{2}}{\rho_{1}} \frac{\gamma_{1}}{\gamma_{2}}\right)^{\frac{1}{2}}
$$

The weakness of correlations along the line of (1) lies in the fact that none of the 'independent' variables describes the intrinsic compressibility of the flow as introduced in $\S 1 . M_{1}$ itself is not an intrinsic-compressibility parameter since a flow with large $M_{1}$ may also have $U_{2} \approx U_{1}$, thus making the velocity difference low subsonic with respect to either speed of sound. Such is Case 1 of the present experiments.

Below we examine ways to express compressibility in more adequate fashion. In particular, we follow and enlarge on the work of Bogdanoff (1983), who introduced a reference Mach number $M^{+}$in a coordinate system moving with the instability waves.

If the shear layer were reduced to an infinitely thin, wavy interface between the two free streams, i.e. a vortex sheet, we could describe its compressibility in a frame of reference in which the wave is stationary. In other words, instead of using the Mach numbers in the laboratory coordinate system, we use those in a coordinate system moving with the wave speed, thus those that the wave itself 'feels'. The vortex sheet in compressible flow, in the limit of small disturbance, is a simple and elegant problem that has been solved analytically (Landau 1944; Pai 1954; Miles 1958; and others). To test our concept in this simple flow, we performed our own 
(a)

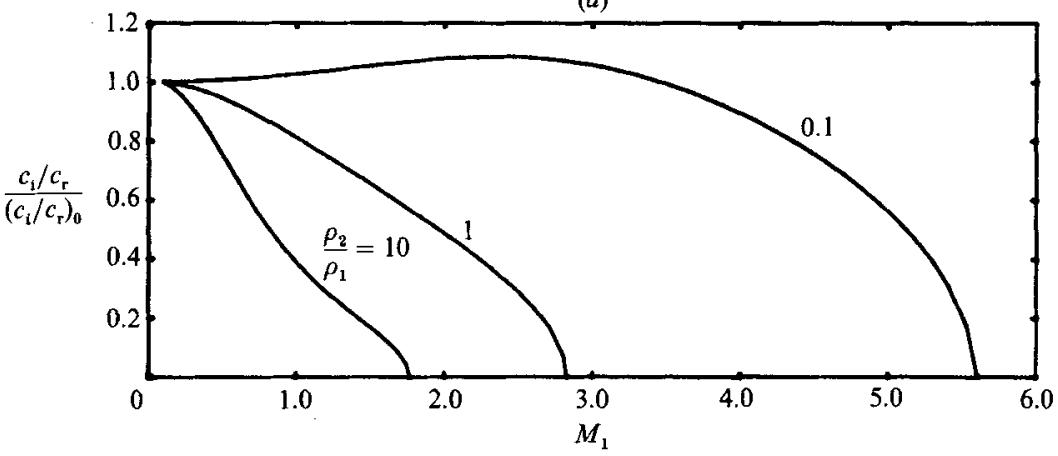

(b)

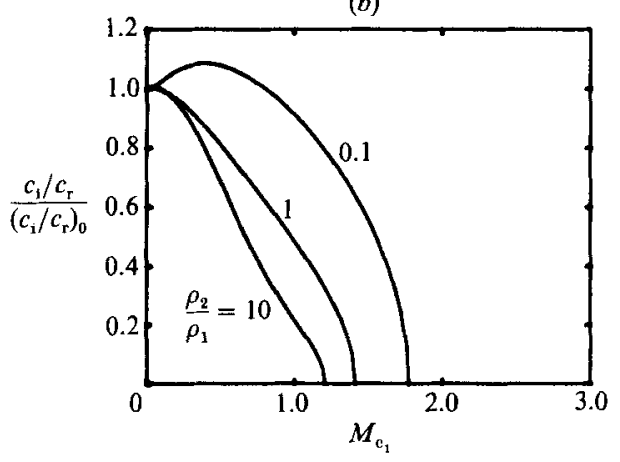

Figure 10. Vortex-sheet amplification rate, normalized by incompressible value, for $M_{2}=0$, $\gamma_{2} / \gamma_{1}=1$, and $\rho_{2} / \rho_{1}=0.1,1,10$. (a) Plotted versus $M_{1} ;(b)$ plotted versus $M_{\mathrm{e}_{1}}$.

compressible vortex-sheet analysis and computation. It is similar to those found in the literature but encompasses a larger range of flow parameters (Papamoschou 1986). Below we highlight the results so obtained.

Consider the temporally developing vortex sheet: in a frame moving with the wave speed $c_{\mathrm{r}}$ the instability grows exponentially in time at a rate $c_{\mathrm{i}}\left(c=c_{\mathrm{r}}+\mathrm{i} c_{\mathrm{i}}\right.$ is the complex phase velocity). The ratio $c_{\mathrm{i}} / c_{\mathrm{r}}$ may be viewed as the spatial amplification rate of the sheet. It is a function of all the variables of the right-hand side of (1). First, we want to examine its dependence on $M_{1}$ for given values of the other parameters. Again, $M_{2}$ is redundant and given by (2).

In the example shown on figure $10(a), U_{2} / U_{1}=0, \gamma_{2} / \gamma_{1}=1$ and $\rho_{2} / \rho_{1}$ takes the values $0.1,1.0$, and 10 . To show the effect of $M_{1}$ most clearly, the amplification rate is normalized to be 1 at $M_{1}=0$. It is seen to decline gradually with increasing $M_{1}$, until it vanishes at some critical value of $M_{1}$. Beyond that value, the vortex sheet is stable, a well-known fact from the above-mentioned vortex-sheet analyses. That critical value depends on $U_{2} / U_{1}, \rho_{2} / \rho_{1}$, and $\gamma_{2} / \gamma_{1}$. Here we see its dependence on $\rho_{2} / \rho_{1}$ only and notice that it is large.

Consider the same example, but correlate against the parameter

$$
M_{\mathrm{e}_{1}}=\frac{U_{1}-c_{\mathrm{r}}}{a_{1}} .
$$

We call it the convective Mach number since it is the Mach number in a frame of reference convecting with the wave speed $c_{\mathrm{r}}$. There is a similarly defined $M_{\mathrm{c}_{2}}$ which, like $M_{2}$, becomes redundant given the other flow parameters. The growth rate versus 


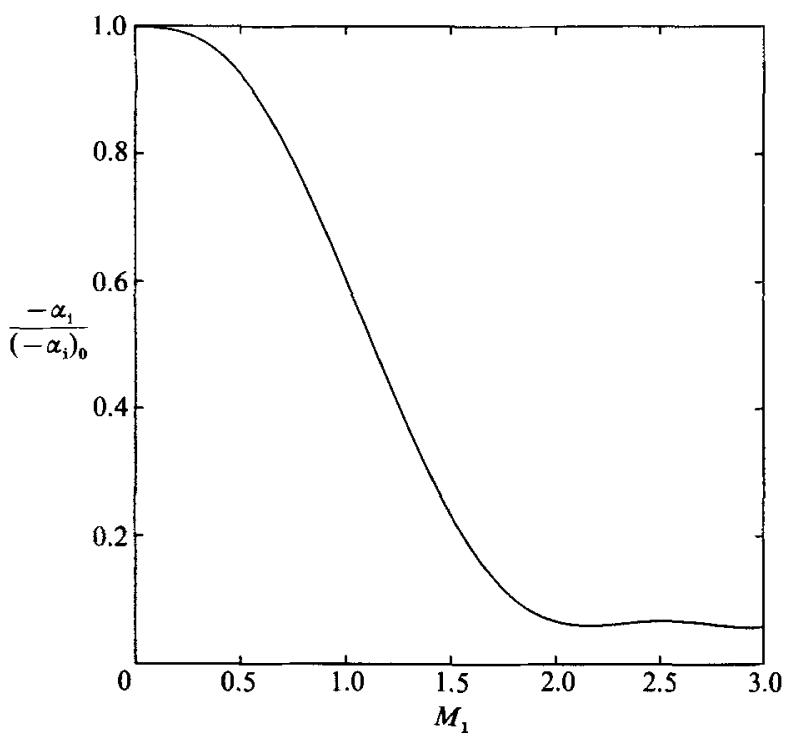

Froure 11. Maximum spatial amplification rate, normalized by its incompressible value, for finite-thickness shear layer as computed by Gropengiesser (1970). $M_{2}=0, \rho_{2} / \rho_{1}=1, \gamma_{1} / \gamma_{2}=1$.

$M_{\mathrm{c}_{1}}$ is shown on figure $10(b)$. One sees the same trends as in the previous correlations versus $M_{1}$, only now the curves for different density ratios are close to each other. Also, the critical value of $M_{c_{1}}$, at which the amplification rate vanishes, is considerably less sensitive to density ratio than is that of $M_{1}$. In a sense, $M_{\mathbf{c}_{1}}$ correlates the amplification rates more 'universally' than does $M_{1}$. Furthermore, our analysis shows that $M_{\mathrm{c}_{2}}$ is close to $M_{\mathrm{c}_{1}}$ and its critical value lies in the same range as that of $M_{\mathrm{c}_{1}}$. Similar unifying qualities of $M_{\mathrm{c}_{1}}$ are seen when $U_{2} / U_{1}$ or $\gamma_{2} / \gamma_{1}$ are varied. Our calculation therefore suggests that, for the vortex sheet, compressibility is more properly described in a coordinate system moving with the wave, than in a stationary one.

For the finite-thickness shear layer, linearized stability analyses are scarce in the literature. Lin (1953) laid the foundation for such analyses, although his is restricted to subsonic disturbances, i.e. disturbances with convective Mach number less than one. The works of Gropengiesser (1970) and of Blumen, Drazin \& Billings (1975) extend to supersonic disturbances. The latter two authors find that the amplification rates decline rapidly with increasing Mach number, starting at subsonic values, but never become zero no matter how large the Mach number. One of Gropengiesser's results is plotted on figure 11, showing the maximum spatial amplification rate $-\alpha_{\mathrm{i}}$ versus $M_{1}$ in a one-stream $\left(M_{2}=0\right)$ shear layer with uniform density. Notable is the flattening of the $-\alpha_{i}$ curve at high Mach numbers. It is important to note that although the above authors did not define a convective Mach number in the sense of (3), terms that contain the convective Mach number implicitly arise naturally in their analyses. The authors did appreciate their importance, since these terms characterize the nature of a disturbance as subsonic, sonic, or supersonic, but did not use them in their correlations.

In the incompressible turbulent case, the discovery of organized large structures and the realization that they are related to the instability of the shear layer led to the idea of a coordinate system moving with the structures. This corresponds to the description of the vortex sheet in a frame moving with the instability wave, as above. 
(a)

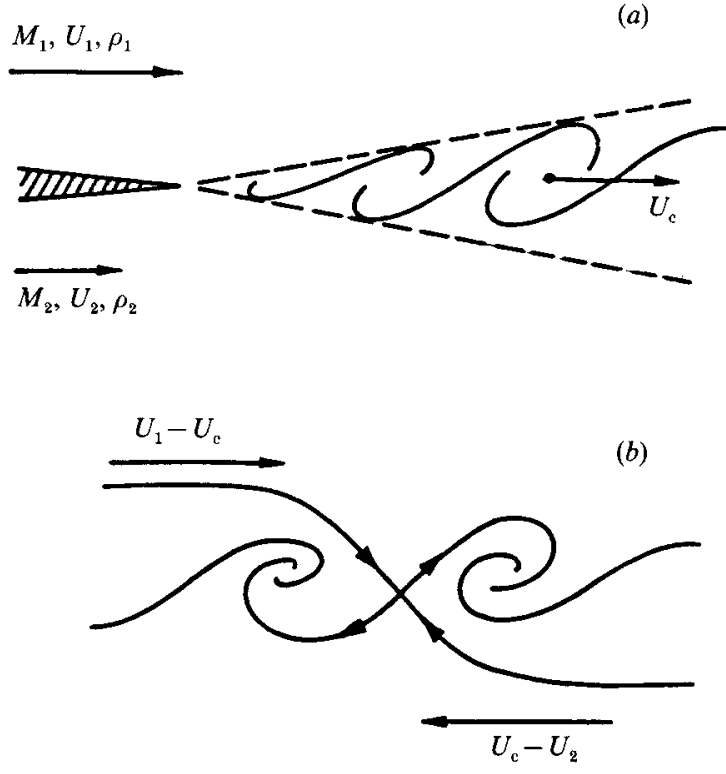

Figure 12. Turbulent shear layer. (a) Stationary frame of reference, $(b)$ convective frame of reference with sketches of streamlines after Coles (1981).

Since large structures are also present in compressible turbulent shear layers, a frame of reference moving with $U_{\mathrm{c}}$, where $U_{\mathrm{c}}$ is the velocity of the dominant waves and structures, appears to be the most suitable one in which to discuss the flow. Accordingly, we define the convective Mach numbers of a turbulent shear layer as follows :

$$
\left.\begin{array}{c}
M_{\mathrm{c}_{1}}=\frac{U_{1}-U_{\mathrm{c}}}{a_{1}}, \\
M_{\mathrm{c}_{2}}=\frac{U_{\mathrm{c}}-U_{2}}{a_{2}}
\end{array}\right\}
$$

An idealized view of the large-scale structure, shown on figure $12(a)$, leads to Bogdanoff's (1983) formulation of a pressure boundary condition by which the convective $U_{\mathrm{c}}$ is calculated. It is implicit in Cole's (1981) sketches of streamlines in the moving coordinates of the large structures, depicted on figure $12(b)$, and was first suggested to us by P. E. Dimotakis (private communication and 1986). In this coordinate system, there is a saddle point between the structures; it is a common stagnation point for both streams, thus implies equality of total pressures in the two streams in that systemn. For equal static pressures and assuming steady flow this results in

$$
\left(1+\frac{\gamma_{1}-1}{2} M_{\mathrm{c}_{1}}^{2}\right)^{\frac{\gamma_{1}}{\gamma_{1}-1}}=\left(1+\frac{\gamma_{2}-1}{2} M_{\mathrm{c}_{2}}^{2}\right)^{\frac{\gamma_{2}}{\gamma_{2}-1}}
$$

For $M_{\mathrm{c}_{1}}$ and $M_{\mathrm{c}_{2}}$ not very large and $\gamma_{1}$ and $\gamma_{2}$ not greatly different, (5) can be approximated by

$$
M_{\mathrm{c}_{2}}=\left(\frac{\gamma_{1}}{\gamma_{2}}\right)^{\frac{1}{2}} M_{\mathrm{c}_{1}}
$$



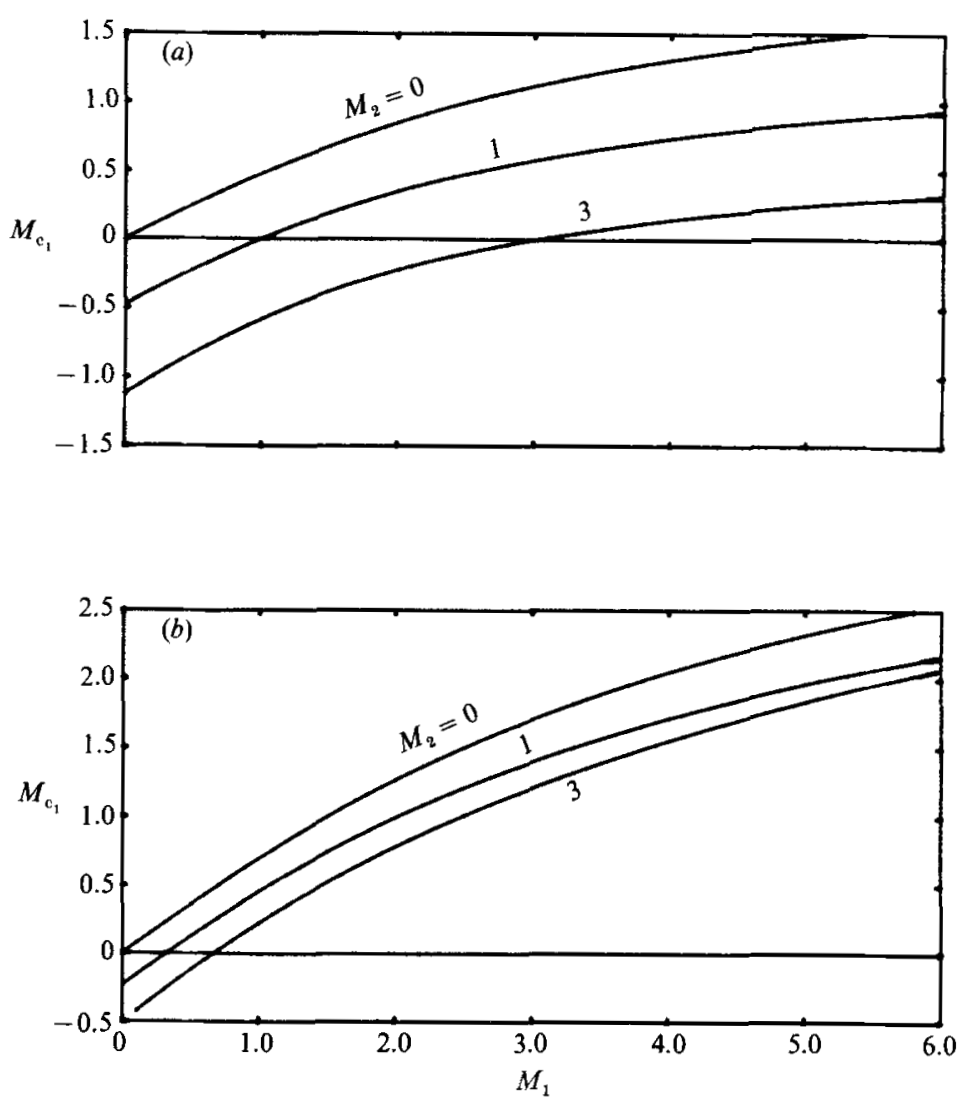

Figure 13. Convective Mach number $M_{\mathrm{c}}$ as a function of $M_{1}$ and $M_{2}$ for uniform total temperature. (a) $\mathrm{N}_{2}-\mathrm{N}_{2}$ combination; (b) He- $\mathrm{N}_{2}$ combination.

which, together with (4), gives

$$
\frac{U_{\mathrm{c}}}{U_{1}}=\frac{1+\frac{U_{2}}{U_{1}}\left(\frac{\rho_{2}}{\rho_{1}}\right)^{\frac{1}{2}}}{1+\left(\frac{\rho_{2}}{\rho_{1}}\right)^{\frac{1}{2}}} .
$$

For $\gamma_{1}=\gamma_{2}, U_{c}$ can be expressed in the form

$$
U_{c}=\frac{a_{2} U_{1}+a_{1} U_{2}}{a_{1}+a_{2}}
$$

which has the form of a speed-of-sound weighted average. It should be pointed out that (7) and (8) are not restricted to compressible flow and are actually more accurate for incompressible flow. From (6) we find that $M_{\mathrm{e}_{1}}$ and $M_{\mathrm{c}_{2}}$ are only $9 \%$ different when $\gamma_{1}=\frac{7}{5}$ and $\gamma_{2}=\frac{5}{3}$ or the reverse. Figure 13 shows the dependence of $M_{\mathrm{c}_{1}}$ on $M_{1}$ and $M_{2}$ for two gas combinations. $M_{\mathrm{c}_{1}}$ has been calculated for uniform total temperature using (4) and (7).

We now hypothesize that the effects of compressibility, together with those of the other parameters, on the turbulent growth rate are more universally expressed in the form

$$
\delta^{\prime}=f\left(\frac{U_{2}}{U_{1}}, \frac{\rho_{2}}{\rho_{1}}, \frac{\gamma_{2}}{\gamma_{1}}, M_{\mathrm{c}_{1}}\right)
$$


The parameters enclosed in the parentheses of (9) uniquely define the free-stream conditions of the layer. The choice of $M_{\mathrm{c}_{1}}$ or $M_{\mathrm{c}_{2}}$ as a compressibility-effect parameter is not critical since the relation between the two is known from (6) and the two are not very different in the present experiments. Here we choose to correlate against $M_{\mathrm{c}_{1}}$. The values of $M_{\mathrm{c}_{1}}$ in our experiments are shown on table 1 . They are based on the free-stream conditions in the region where the turbulent growth rate is measured.

\section{The net effect of compressibility}

To illustrate a basic point, we first plot the experimental growth rates versus $M_{\mathrm{c}_{1}}$ without considering the effects of the other parameters formulated in (9). An inconclusive trend is obtained, depicted on figure 14, in which the growth rates increase at high $M_{\mathrm{c}_{1}}$, contradicting the expectation supported by previous experimental works that compressibility has a stabilizing effect. We thus realize the need to uncouple the effects of $M_{\mathrm{c}_{1}}$ from those of $U_{2} / U_{1}$ and $\rho_{2} / \rho_{1}$. Since $\gamma$ changes only from $\frac{7}{5}$ to $\frac{5}{3}$, we think that the effect of $\gamma_{2} / \gamma_{1}$ is not as significant here as those of the other parameters, although we cannot prove this rigorously,

A straightforward way to estimate the net effect of $M_{c_{1}}$ is to compare the experimentally measured growth rates with those that would have occurred if the flow were incompressible and the velocity and density ratios were kept unchanged. More specifically, we form the ratio

$$
\frac{\delta^{\prime}}{\delta_{0}^{\prime}}=\frac{f\left(\frac{U_{2}}{U_{1}}, \frac{\rho_{2}}{\rho_{1}}, M_{\mathrm{c}_{1}}\right)}{f\left(\frac{U_{2}}{U_{1}}, \frac{\rho_{2}}{\rho_{1}}, M_{\mathrm{c}_{1}}=0\right)}
$$

which will give the isolated effect of $M_{\mathrm{e}_{1}}$ at particular values of velocity ratio and density ratio. Subscript 0 denotes the incompressible value. This constitutes our generalization of the results obtained by Bogdanoff (1983).

In order to normalize the growth rate according to (10), we need an approximate model for the growth rate of the incompressible shear layer over a wide range of velocity and density ratios. In creating such a model, we assume that the main effect of the density ratio is to determine the convective velocity of the structures $U_{\mathbf{c}}$ as given by (7). We conjecture that in a frame of reference moving with $U_{\mathrm{c}}$ the growth rate is simply proportional to $\Delta U=U_{1}-U_{2}$, thus the density ratio drops out of the picture in that frame. This reasoning leads to the simple expression

$$
\delta_{0}^{\prime} \sim \frac{\Delta U}{U_{\mathrm{c}}} .
$$

For uniform density, from (7) we have $U_{\mathrm{c}}=\frac{1}{2}\left(U_{1}+U_{2}\right)$ and (11) takes the familiar form

$$
\delta_{0}^{\prime} \sim \frac{U_{1}-U_{2}}{U_{1}+U_{2}}
$$

For the visual thickness (11) becomes, using (7),

$$
\delta_{\mathrm{vis}, \mathbf{0}}^{\prime}=0.17 \frac{\Delta U}{U_{\mathrm{c}}}=0.17 \frac{\left[1-\frac{U_{2}}{U_{1}}\right]\left[1+\left(\frac{\rho_{2}}{\rho_{1}}\right)^{\frac{1}{2}}\right]}{1+\frac{U_{2}}{U_{1}}\left(\frac{\rho_{2}}{\rho_{1}}\right)^{\frac{1}{2}}},
$$




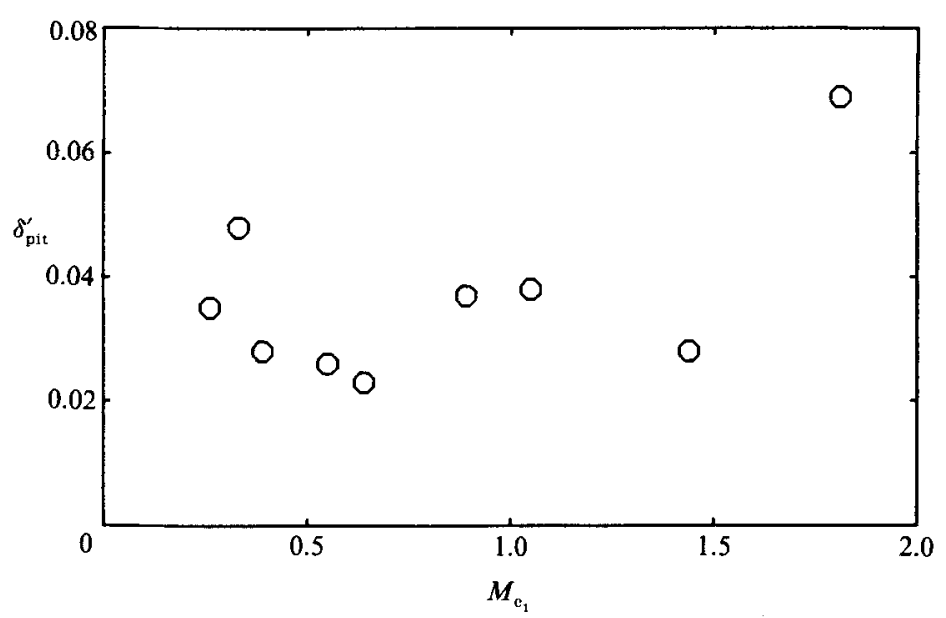

Figure 14. Pitot-thickness growth rate versus $M_{\mathrm{c}_{1}}$.

the constant 0.17 having been obtained experimentally by Brown \& Roshko (1974). A generalized version of (13), obtained by a similar hypothesis, was first proposed by Brown (1974). Dimotakis (1986) used geometrical arguments to derive the entrainment and growth of the turbulent region and found an expression for the growth rate similar to $(13)$.

Although the present derivation of (13) is not rigorous, its growth-rate prediction is in fairly good agreement with experimentally observed visual rates of subsonic shear layers, as shown in figure 15. To compare with the present results of Pitotthickness growth we assume, as explained in $\S 5$, that $\delta_{\text {pit }, 0}^{\prime}=0.8 \delta_{\text {vis, } 0}^{\prime}$. From (13) we then have

$$
\delta_{\mathrm{pit}, 0}^{\prime}=0.14 \frac{\left[1-\frac{U_{2}}{U_{1}}\right]\left[1+\left(\frac{\rho_{2}}{\rho_{1}}\right)^{\frac{1}{2}}\right]}{1+\frac{U_{2}}{U_{1}}\left(\frac{\rho_{2}}{\rho_{1}}\right)^{\frac{1}{2}}} .
$$

The ratio $\delta_{\text {pit }}^{\prime} / \delta_{\text {pit }, 0}^{\prime}$, called here the normalized growth rate, is now formed according to $(10)$, where $\delta_{\text {pit }}^{\prime}$ is the experimentally obtained growth rate and $\delta_{\text {pit, } 0}^{\prime}$ is the incompressible Pitot growth rate, obtained from (14), at the same values of $U_{2} / U_{1}$ and $\rho_{2} / \rho_{1}$ as the experimental one. The normalized growth rate is plotted versus $M_{\mathrm{c}_{1}}$ in figure 16 . Its values collapse roughly onto one curve, which suggests that the growth rate of a compressible shear layer might be approximately expressed in the form

$$
\frac{\delta^{\prime}}{\delta_{0}^{\prime}} \approx C\left(M_{\mathrm{e}_{1}}\right)
$$

where $C$ is a universal function valid over a wide range of $U_{2} / U_{1}$ and $\rho_{2} / \rho_{1}$. We must be cautious about such a statement, however, in view of the limited number of measurements and the experimental error involved. The accuracy of the incompressible model of the shear layer must also be taken into consideration.

What is remarkable about the curve of figure 16 is the drastic reduction of the normalized growth rate with increasing $M_{\mathrm{c}_{1}}$, until $M_{\mathrm{c}_{1}}$ reaches about 0.8 . Beyond that point, it stays fairly constant, at a value approximately one fifth of the incompressible one. The normalized growth rate declines gradually with increasing 


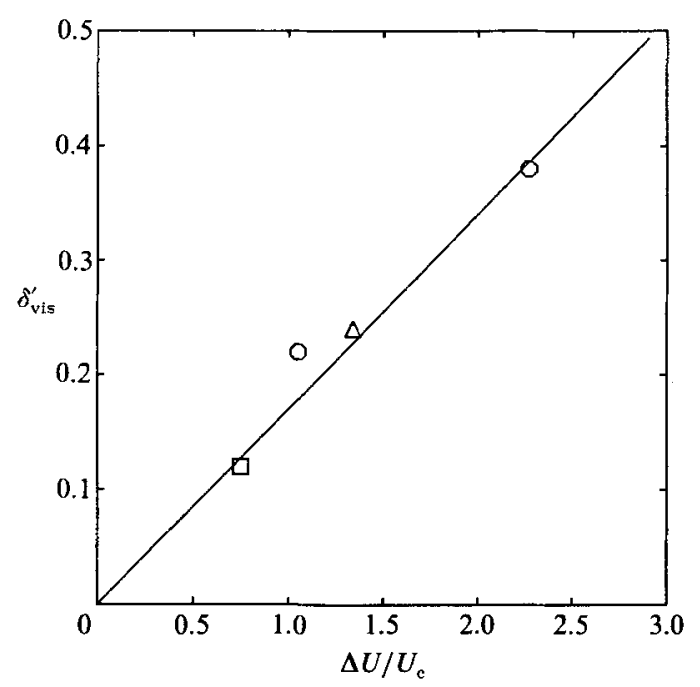

Figure 15. Model for visual growth of incompressible shear layer. Circle and square data points from Brown \& Roshko (1974) for $\rho_{2} / \rho_{1}=7$ and $\frac{1}{7}$ respectively; triangle from Dimotakis \& Brown (1976) for $\rho_{2} / \rho_{1}=1$.

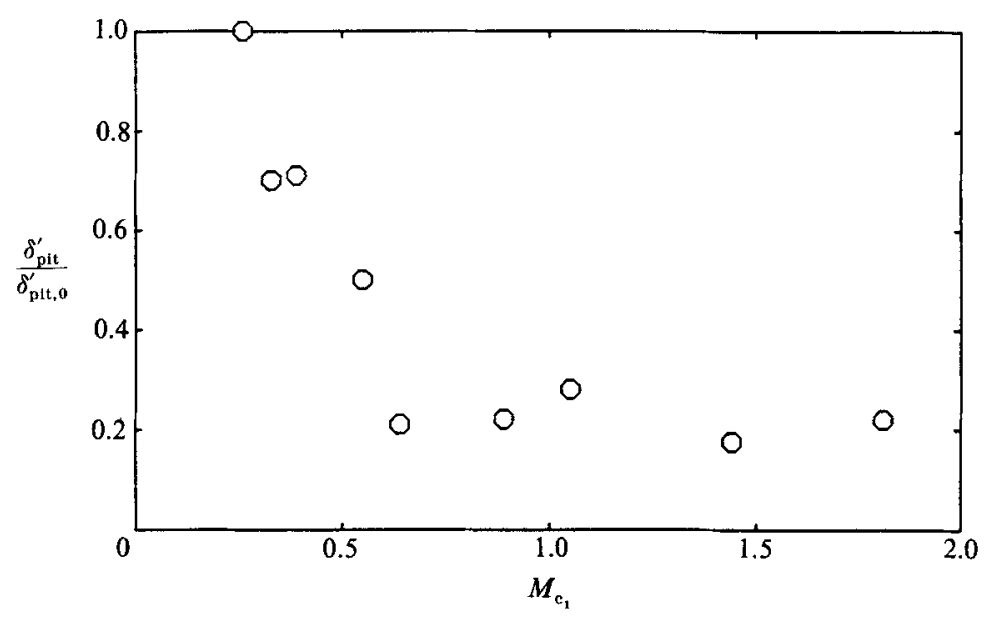

Fiavre 16. Normalized Pitot-thickness growth versus $M_{\mathrm{e}_{1}}$.

$M_{\mathrm{c}_{1}}$, starting at subsonic values of $M_{\mathrm{c}_{1}}$. This contrasts with some earlier views that the decline of growth rate would come abruptly, immediately after some relative Mach number becomes supersonic (Cosner 1976). The general features of this curve, particularly the flattening at large values of $M_{c_{1}}$, are similar to those of Gropengiesser's curve (figure 11).

Assuming that (15) is approximately valid, at a given value of $M_{c_{1}}$ the effects of density ratio and velocity ratio on the growth rate are similar to those in an incompressible shear layer, given by (14): the growth rate is smaller when the heavier gas is on the high-speed side and greater when the heavier gas is on the low-speed side; the growth rate increases with decreasing velocity ratio, i.e. with increasing free-stream-velocity difference.

The same type of correlation used with the present growth rates can be applied to the growth rates measured by previous investigators. The experimental data are 


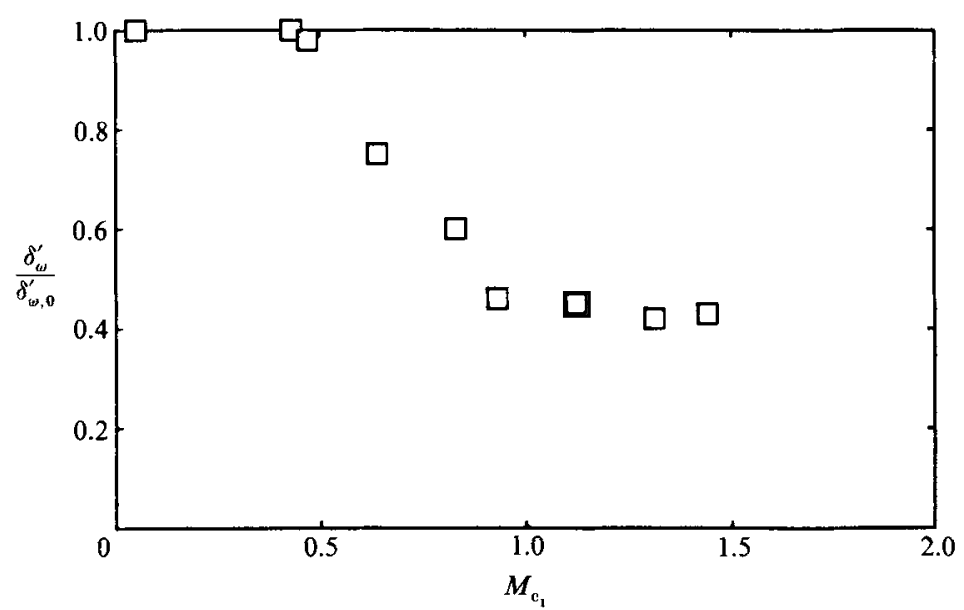

FigURE 17. Normalized vorticity-thickness growth versus $M_{c_{1}}$. Original data obtained by other investigators as compiled in figure 1 of Bogdanoff (1983).

taken from Maydew \& Reed (1963), Sirieix \& Solignac (1966), Birch \& Eggers (1973), and Ikawa \& Kubota (1975). These investigators made probe measurements of vorticity thickness $\left(\delta_{\omega}\right)$ in shear layers with $M_{2}=0$ and both gases air. We form the ratio $\delta_{\omega}^{\prime} / \delta_{\omega, 0}^{\prime}$ using (13) with the constant changed to 0.085 . That change stems from the fact that in incompressible homogeneous shear layers the vorticity thickness is about half of the visual thickness (Brown \& Roshko 1974). There is no evidence, however, to suggest that the same relation holds for layers with density difference. The result is depicted on figure 17 where we see that the normalized growth rate versus $M_{\mathrm{c}_{1}}$ exhibits the same qualitative behaviour as the current one. Quantitatively, the major difference is that the levelling off of the curve at $M_{\mathrm{c}_{1}}>1$ occurs at a normalized growth rate around 0.4 , as opposed to 0.2 in the present experiments. Also, the decrease in normalized growth rate starts at $M_{\mathrm{c}_{\mathrm{s}}}=0.4$, rather than at $M_{\mathrm{e}_{1}}=0.25$ in our cases. The possible dependence of the ratio $\delta_{\nu} / \delta_{\mathrm{pit}}$ on density ratio and Mach number may account for this difference.

\section{Discussion}

We hope that with this experimental study we have achieved two main goals: to produce useful data for scientific and engineering applications; and to put the issue of compressibility in a perspective that captures the physics of the flow, thus enhances our understanding of its effects. The latter point is crucial because the effect of compressibility on the spreading rate of the turbulent shear layer is more subtle than the effects of velocity and density ratios. Still, we have difficulty in formulating a solid physical explanation as to why the growth rate behaves as it does without getting into the details of some complex analysis. Below, we offer some simple thoughts that may provide some preliminary insight into the behaviour of these complex flows.

It is interesting that the growth-rate reduction starts at subsonic values of $M_{c_{1}}$ and is evidently completed before $M_{c_{1}}$ becomes supersonic (figure 15). This implies that compressibility takes effect before any shock or expansion waves appear in the flow, in the convective frame of reference. A similar trend is seen from the vortex-sheet calculations (figure $10 \mathrm{~b}$ ). In the vortex sheet, the solution for the perturbation 
(a)

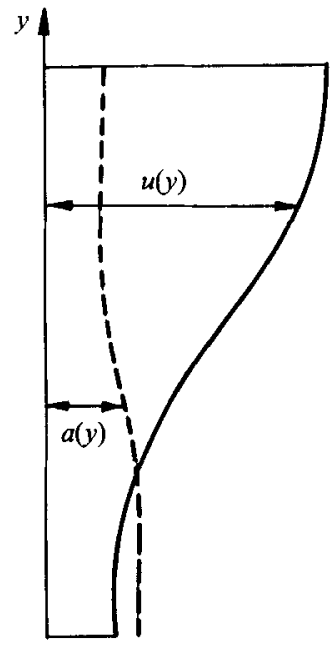

(b)

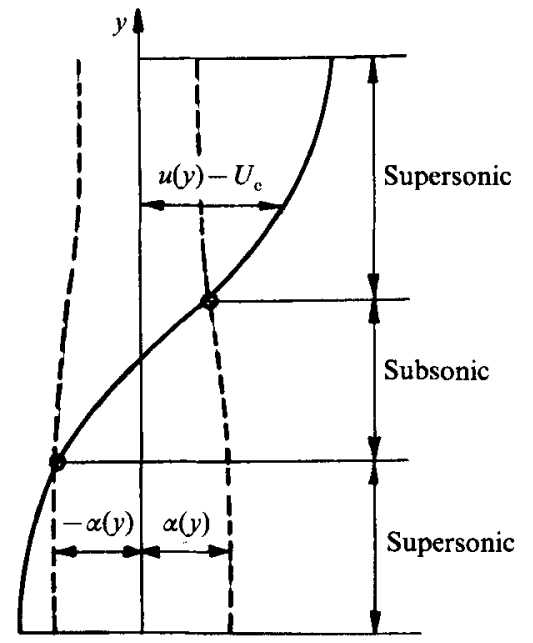

Figure 18. Velocity and speed-of-sound profiles for illustration purposes. (a) Stationary frame of reference; $(b)$ convective frame of reference, with disturbance characterization.

potential shows its amplitude decaying exponentially with distance away from the sheet at a certain rate (the reader may wish to consult the relevant references listed in $\S 6$ ). That decay rate depends strongly on $M_{\mathrm{c}_{1}}$ : it is generally largest at $M_{\mathrm{c}_{1}}=0$ and decreases with increasing $M_{c_{1}}$. At and beyond the critical value of $M_{c_{1}}$ (the value at which the sheet is stabilized), the decay rate is zero, meaning that the perturbation propagates unattenuated (radiates) away from the sheet. The decrease of amplification rate is thus associated with the disturbance penetrating further into the surrounding fluid. We propose to interpret that in the following fashion: as $M_{\mathrm{c}_{1}}$ increases, a disturbance acts less in perturbing the flow in the vicinity of the sheet, and thus the sheet itself, and more in perturbing the flow away from the sheet. Conceptually, a similar mechanism may be responsible for the gradual growth reduction in the turbulent case. Morkovin (1987) studied the boundary-layer transition at supersonic speeds and noted that at supersonic convective Mach numbers energy is radiated away from the layer. He argued that this causes energy removal from potential pairing of spanwise vortices, impeding the process of amalgamation. Whether lack of amalgamation is responsible for our small growth rates is not clear, but it is reasonable to expect that energy removal from the shear layer would slow down all interactions responsible for growth. Our present data suggest that this energy removal starts at subsonic $M_{\mathrm{c}_{1}}$ and gets stronger as $M_{\mathrm{c}_{1}}$ increases, as reflected in our growth-rate-versus- $M_{\mathbf{c}_{1}}$ curve (figure 16 ).

That there is a distinction between a vortex sheet and a shear layer of finite thickness seems elementary. It is in that obvious difference that we see a fundamental feature of the finite-thickness shear layer that perhaps has not been fully appreciated yet. This feature is illustrated on figure 18, where distributions of velocity and speed of sound are depicted in stationary and convective frames of reference. In this example, $M_{\mathrm{c}_{1}}$ and $M_{\mathrm{c}_{2}}$ are both supersonic. There is a portion of the flow near the centre of the layer where the local velocity, relative to the convective velocity, is subsonic. It is evident that the extent of the 'subsonic' sublayer will decrease with increasing $M_{\mathrm{c}_{1}}$ and $M_{\mathrm{e}_{2}}$, but will never vanish. The fact that in a compressible shear 
layer there is always an imbedded subsonic region, thus a region potentially dominated by subsonic-type instabilities, is, in our view, significant. Viewed in the convective frame, this is a region where a disturbance can propagate upstream. Mack (1984) and Morkovin (1987) stress that such upstream communication is essential for instability at supersonic speeds. Lees \& Gold (1965), in their analysis of supersonic wakes, also realize the existence of an intrinsically subsonic region even though the disturbance is supersonic with respect to the free stream. We believe that this feature is connected to the fact that the shear layer remains unstable no matter how large the Mach number.

As noted early in $\$ 6$, our analyses and discussions have so far been limited to twodimensional disturbances. It is possible that oblique disturbances, i.e. disturbances that propagate at an angle $\beta$ to the mean-flow direction, exist in compressible shear layers. In the analytical works of Lessen, Fox \& Zien (1965) and of Gropengiesser it is found that the shear layer becomes more unstable as $\beta$ increases. Bogdanoff (1983) proposes that in the turbulent shear layer the effect of $\beta$ can be taken into account by defining an effective convective Mach number

$$
M_{\mathrm{c}_{1, \mathrm{eff}}}=M_{\mathrm{c}_{1}} \cos \beta
$$

One can easily appreciate the profound changes that a large value of $\beta$ may bring: a flow with $M_{\mathrm{c}_{1}}>1$ may have $M_{\mathrm{c}_{1, \text { eff }}}<1$. Since the disturbance is now characterized by $M_{\mathrm{c}_{1, \text { eff }}}$ rather than by $M_{\mathrm{e}_{1}}$, the entire flow is intrinsically subsonic, thus more unstable than one would predict using two-dimensional arguments.

We speculate that the structures in our experiments are quasi-two-dimensional: the schlieren photographs, which give a spanwise-integrated view of the shear layer, reveal distinct structures. Since the shear layer has an aspect ratio of about 10 , we think it is unlikely that such distinct images would appear unless these structures were organized spanwise. Admittedly, this is a weak argument that needs to be substantiated by experiments. We must also consider the possibility of a spanwise structure superimposed on the larger one, such as exists in subsonic shear layers, with primary structure that is coherent spanwise (Konrad 1976; Breidenthal 1981 ; Bernal 1981).

Finally, we would like to comment on the adverse streamwise pressure gradient occurring in the near field (the growth-rate measurements were done in the far field, as detailed in $\S 3$ ) present in our experiments. Although its dependence on the flow parameters is not well understood, we suspect that its severity increases with increasing trailing-edge Mach number. There may thus exist a practical limit as to how high a Mach number one can sustain in the far field, downstream of the pressuregradient region. This would also translate into a limit for the convective Mach number. On the other hand, little research has been done on the dependence of the pressure gradient on channel geometry. It could possibly be counteracted, or at least delayed, by a test-section shape that is more sophisticated than the current one.

This work was initiated and supported mainly by a research grant from the Rockwell International Corporation Trust; additional support was obtained from the US Navy Office of Naval Research as part of Contract No N00014-85-K-0646. 


\section{REFERENCES}

BaILEy, H. A. \& Kuethe, A. M. 1957 Supersonic mixing of jets and turbulent boundary layers. WADC Tech. Rep. 57-402.

Bernat, L. P. 1981 The coherent structure of turbulent mixing layers. Ph.D. thesis, California Institute of Technology.

Birch, S. L. \& EGgERS, J. M. 1973 A critical review of the experimental data for developed free turbulent shear layers. NASA SP-321, pp. 943-949.

Blumen, W., Drazin, D. G. \& Billings, D. F. 1975 Shear layer instability of an inviscid compressible fluid. Part 2. J. Fluid Mech. 71, 305-316.

Bogdanoff, D. W. 1983 Compressibility effects in turbulent shear layers. AIAA J. 21, 926-927.

BRADShaw, P. 1966 The effect of initial conditions on the development of a free shear layer. J. Fluid Mech. 26, 225-236.

BREID enthal, R. 1981 Structure in turbulent mixing layers and wakes using a chemical reaction. J. Fluid Mech. 109, 1-24.

BRown, G. L. 1974 The entrainment and large structure in turbulent mixing layers. Fifth Australian Conf. on Hydraulics and Fluid Mechanics, Christchurch, New Zealand, pp. 352-359.

Brown, G. L. \& Roshro, A. 1974 On density effects and large structures in turbulent mixing layers. J. Fluid Mech. 64, 775-781.

Chinzei, N., Masuya, G., Komuro, T., Murakami, A. \& Kudou, K. 1986 spreading of twostream supersonic mixing layers. Phys. Fluid 29, 1345-1347.

Coles, D. 1981 Prospects for useful research on coherent structure in the turbulent shear flow. Proc. Indian Acad. Sci. 4, 111-127.

CosNer, R. R. 1976 Experiments on thin airfoils spanning a transonic shear flow. Ph.D. thesis, California Institute of Technology.

Demetriades, A. \& Brower, T. L. 1982 Experimental study of transition in a compressible free shear layer. AFOSR-TR 83-0144.

Diмotakis, P. E. 1986 Two-dimensional shear-layer entrainment. $A I A A$ J. 24, 1791-1796.

Dimotakis, P. E. \& Brown, G. L. 1976 The mixing layer at high Reynolds number: large structure dynamics and entrainment. J. Fluid Mech. 78, 535-560.

Dutron, J. C., Mikkelsen, C. D. \& Addy, A. L. 1982 A theoretical and experimental investigation of the constant-area, supersonic ejector. AIAA J. 20, 1392-1400.

Gropengiesser, H. 1970 Study of the stability of boundary layers in compressible fluids. NASA $T T-F-12$, p. 786.

IKaWA, H. \& KUвотA, T. 1975 Investigation of supersonic turbulent mixing with zero pressure gradient. AIAA J. 13, 566-572.

KonRAD, J. H. 1976 An experimental investigation of mixing in two-dimensional turbulent shear flows with applications to diffusion-limited chemical reactions. Ph.D. thesis, California Institute of Technology.

LANDAU, L. 1944 Stability of tangential discontinuities in compressible fluid. Dokl. Akad. Nauk. SSSR 44, 139-141.

LeEs, L. \& GoLd, H. 1965 Stability of laminar wakes. In Fundamental Phenomena in Hypersonic Flow, pp. 310-337. Cornell University Press.

Lfssen, M., Fox, J. A. \& ZIEN, H. M. 1965 On the inviscid stability of the laminar mixing of two parallel streams of a compressible fluid. J. Fluid Mech. 23, 355-367.

Lrs, C. C. 1953 On the stability of the laminar mixing region between two parallel streams in a gas. NACA TN 2887.

MACK, L. M. 1984 Boundary-layer linear stability theory. AGARD Rep. 709.

Maydew, R. C. \& ReEd, J. F. 1963 Turbulent mixing of compressible shear layers. AIAA J. I, $1443-1444$

Miles, J.W. 1958 On the disturbed motion of a plane vortex sheet. J. Fluid Mech. 4, $538-552$. 
Morkovis, M. V. 1987 Transition at hypersonic speeds. NASA CR 178315, ICASE Interim Rep. 1.

OERTEL, H. 1979 Mach wave radiation of hot supersonic jets investigated by means of the shock tube and new optical techniques. Proc. 12th Intl Symp. of Shock Tubes and Waves. Jerusalem. pp. 266-275.

Ortwerth, P. J. \& Shine, A. J. 1977 On the scaling of plane turbulent shear layers. $A F W L-T R$ $77-118$.

PAI, S. I. 1954 On the stability of a vortex sheet in an inviscid compressible fluid. J. Aero. Sci. 21, $325-328$.

Papayoschov, D. 1986 Experimental investigation of heterogeneous compressible shear layers. Ph.D. thesis, California Institute of Technology.

Papamoschoc, D. \& Roshko, A. 1986 Observations of supersonic free shear layers. AIAA-860162 .

Rott, N. \& Crabtree. L. F. 1952 Simplified laminar boundary-layer calculation for bodies of revolution and for yawed wings. J. Aero. Sci. 19, 553-565.

Shackleford, W. L., Witte, A. B., Broadwell, J. E., Trost, J. E. \& Jacobs, T. A. 1973 Experimental studies of chemically reactive flow in supersonic free jet mixing layers. $A I A A$ 73-640.

Sirieix, M. \& Solignac', J. L. 1966 Contribution a l'etude experimentale de la couche de melange turbulent isobare d'un ecoulement supersonique. Symposium on Separated Flow, AGARD Conf. Proc. 4, 241-270.

Sprncer, B. W. \& Jonts, B. G. 1971 Statistical investigation of pressure and velocity fields in the turbulent two-stream mixing layer. $A I A A-71-613$. 